\title{
A Very Rare Adult Case with Neuroblastoma
}

\author{
Fatih Selcukbiricik Deniz Tural Nihal Esatoglu \\ Sinem Kocak Nil Molinas Mandel
}

Department of Medical Oncology, Cerrahpasa Faculty of Medicine, University of Istanbul, Istanbul, Turkey

\section{Key Words}

Adult neuroblastoma Chemotherapy

\begin{abstract}
We report a 53-year-old male patient who underwent paravertebral mass excision at the D10-11-12 vertebral levels in 2007. The histopathological evaluation of the mass showed the presence of neuroblastoma. The patient was diagnosed with stage IV neuroblastoma. He received 6 courses of chemotherapy and exhibited a stable course until March 2010. When he was reevaluated in March 2010, progression in the metastatic lesion as well as local recurrence was detected. The patient, who was restarted on chemotherapy, developed progressive weakness and loss of sensation of the lower extremity. The neurosurgical investigation revealed an irreversible loss in motor functions. The patient is currently on symptomatic treatment.
\end{abstract}

\section{Introduction}

Neuroblastoma (NB) is the most common extracranial solid tumor in childhood that originates from the neural crest. The mean age of diagnosis is 2 years, with $35 \%$ of the cases occurring before the age of 1 year and the remaining occurring before 10 years of age. NB affects children in the first year of life and is very rare among adolescents and young adults. The most common site of involvement for NB is the abdomen (60\%), followed by the thorax (15\%), pelvis (5\%) and the cervical sympathetic chain (5\%). Approximately $20 \%$ of all NBs originate from the posterior mediastinum. The treatment of NB is surgical chemotherapy or radiotherapy. Currently, there is no standard treatment protocol for the adult NB. The treatment protocol established for children remains inappropriate for adults and particularly, localized disease involves a more aggressive course in adults compared to children. In NB, the treatment should be performed according to the pediatric guidelines because no treatment recommendations exist for the treatment of NB in adults. 


\section{Case Report}

A 53-year-old male patient underwent paravertebral mass excision at the D10-11-12 vertebral levels in 2007 (fig. 1a, b). The histopathological evaluation of the mass showed the presence of NB (fig. 2a, b). Neuron-specific enolase was $>370 \mathrm{ng} / \mathrm{ml}$. Upon presentation with the complaints of pain in the right hip and claudication after 1 year, a mass lesion with indefinite edges that surrounded the right iliac bone and led to the destruction of cortex was detected. Tru-cut biopsy obtained from the mass was consistent with metastatic NB.

The patient was diagnosed with stage IV NB. Combination chemotherapy with a CADO/CDDPVP16 regimen according to the pediatric protocol was started with cyclophosphamide (CPM 1,500 $\mathrm{mg} / \mathrm{m}^{2}$ per cycle), doxorubicin (AD $60 \mathrm{mg} / \mathrm{m}^{2}$ per cycle), vincristine ( $2 \mathrm{mg}$ per cycle), cisplatin (CDDP $100 \mathrm{mg} / \mathrm{m}^{2}$ per cycle), and etoposide (VP16 $500 \mathrm{mg} / \mathrm{m}^{2}$ per cycle) with G-CSF. Treatment consisting of D1 adriamycin $75 \mathrm{mg} / \mathrm{m}^{2}$, D2 cyclophosphamide $1,800 \mathrm{mg} / \mathrm{m}^{2}$, D1 vincristine $2 \mathrm{mg}$ and D1-5 ifosfamide $1,800 \mathrm{mg} / \mathrm{m}^{2}$, D1-5 etoposide $100 \mathrm{mg} / \mathrm{m}^{2}, \mathrm{D} 1-5$ Mesna $1,800 \mathrm{mg} / \mathrm{m}^{2}$ was planned for the patient. Upon detection of progression in the size of the mass detected in the pelvis in the patient, who received this therapy for 4 courses, D1 cisplatin $80 \mathrm{mg} / \mathrm{m}^{2}, \mathrm{D} 1-3$ etoposide $100 \mathrm{mg} / \mathrm{m}^{2}$ were started concomitantly with radiotherapy. The patient, who received 6 courses of chemotherapy, exhibited a stable course until March 2010. When he was reevaluated in March 2010, progression in the metastatic lesion as well as local recurrence was detected. The patient, who was restarted on chemotherapy, developed progressive weakness and loss of sensation of the lower extremity. The neurosurgical investigation revealed an irreversible loss in motor functions. The patient is currently on symptomatic treatment.

\section{Discussion}

NB is the most common extracranial solid tumor in childhood that originates from the neural crest. The mean age of diagnosis is 2 years with $35 \%$ of the cases occurring before the age of 1 year and the remaining occurring before 10 years of age [1]. They represent $8-10 \%$ of all childhood cancers. NB affects children in the first year of life and is very rare among adolescents and young adults. $\mathrm{NB}$, ganglioneuroblastoma and ganglioneuroma are the tumors that originate from the primordial neural crest cells. NB involves 3 classical histopathological types by differentiation and maturation. These include the NB, ganglioneuroblastoma and ganglioneuroma. While most cases have a nonestablished etiology of NB, environmental factors appear to be involved. The most common site of involvement for NB is the abdomen (60\%), followed by the thorax (15\%), pelvis (5\%) and the cervical sympathetic chain (5\%) [2]. Approximately $20 \%$ of all NBs originate from the posterior mediastinum $[3,4]$. The symptoms of the disease show variability based on the site of involvement; however, they most commonly include the manifestations of compression due to the mass and bone pain due to metastasis.

NB may originate from any site in the sympathetic nervous system. The site of the primary tumor shows variability based on the age of the patients at the time of diagnosis. While a majority of the primary tumors are of intra-abdominal localization (65\%), in children below 1 year of age, the tumors involve the intra-thoracic region and the neck. There is no specific localization observed in adults. Primary tumor may not be detected in approximately $1 \%$ of the cases. NBs can metastasize widely through both lymphatic and vascular routes. The most common sites of metastasis are bone marrow, bones, lymph nodes, liver, skin, and testes. There is a greater frequency of metastases among adolescents in unusual parenchyma of the lung or brain, which are affected more frequently in other malignancies that are typical among adults. 
In contrast to the malignant forms, ganglioneuroma is more common among adults. Ganglioneuroma is a benign tumor and is usually asymptomatic. The symptoms usually occur due to the compression created by the tumor. Due to the vasoactive intestinal polypeptides it secretes, diarrhea, hypertension, hypokalemia or masculinization may be observed rarely $[5,6]$. Adolescents more frequently have an advanced stage of the disease, a low excretion of urinary catecholamines, absence of biological abnormalities and chronic but fatal disease [7].

Ganglioneuroma may rarely include the components of ganglioneuroblastoma, NB and feochromocytoma and be transformed to the malignant peripheral nerve sheath tumor [8]. Since it may be radiologically mixed with many tumors, it is quite difficult to establish the diagnosis postoperatively. Therefore, these masses can mostly be diagnosed postoperatively by histopathological investigation.

Compared to NBs occurring in children, NBs observed in adults occur more frequently as an advanced-stage and metastatic disease [9].

The prognosis of the patients with NB depends on numerous variables; the most important of them are age of the patient at diagnosis and the stage of the tumor, DNA pyloidi, molecular signer, histology, and chromosome 1p, 11q, 17g aberration. The low age at diagnosis and the low stage are 2 important favorable prognostic features.

The treatment of NB is surgical chemotherapy or radiotherapy. Currently, there is no standard treatment protocol for adult NB. The treatment protocol established for children remains inappropriate for adults and particularly, localized disease involves a more aggressive course in adults compared to children. In NB, the treatment should be performed according to the pediatric guidelines because no treatment recommendations exist for the treatment of NB in adults $[10,11]$.

Although currently there are no standard treatment guidelines for patients with NB in adulthood, complete surgical resection is sufficient for low risk and nonmetastatic tumors, but combination therapy with chemotherapy and radiotherapy is necessary for disseminated and recurrent diseases. The treatment includes vincristine 1.5 $\mathrm{mg} / \mathrm{m}^{2} /$ dose/day, etoposide $150 \mathrm{mg} / \mathrm{m}^{2} /$ dose/day, carboplatin $170 \mathrm{mg} / \mathrm{m}^{2} /$ dose $/$ day or cyclophosphamide $200 \mathrm{mg} / \mathrm{m}^{2} /$ dose/day, Mesna $3 \times 40 \mathrm{mg} / \mathrm{m}^{2} /$ dose, etoposide 60 $\mathrm{mg} / \mathrm{m}^{2} /$ dose/day, cisplatin $20 \mathrm{mg} / \mathrm{m}^{2} /$ dose/day or vincristine $0.05 \mathrm{mg} / \mathrm{kg} /$ dose $/$ day, DTIC (Dacarbazine) $7 \mathrm{mg} / \mathrm{kg} /$ dose $/$ day, ifosfamide $50 \mathrm{mg} / \mathrm{kg} /$ dose $/$ day, \%100 Mesna 50 $\mathrm{mg} / \mathrm{kg} /$ dose/day, adriamycin $1 \mathrm{mg} / \mathrm{kg} /$ dose/day.

Treatment was comprised of high-dose cyclophosphamide (at a dose of $140 \mathrm{mg} / \mathrm{kg}$ ), topotecan (at a dose of $8 \mathrm{mg} / \mathrm{m}^{2}$ ), and vincristine (at a dose of $0.067 \mathrm{mg} / \mathrm{kg}$ or $2 \mathrm{mg} / \mathrm{m}^{2}$, whichever was lower; maximum dose, $2 \mathrm{mg}$ ) (HD-CTV) at refractory or recurrent NB. Response to HD-CTV as salvage therapy is significantly less likely in adolescents/adults and in children with NB that is persistent or progressing on treatment rather than newly recurrent off treatment. These findings are broadly applicable and should be considered when designing, and interpreting the results of, phase 2 studies [12].

Stage III and IV tumors are often initially treated with chemotherapy followed by tumor resection. Because of a lack of experience in adults, the treatment followed pediatric protocols. The most frequently used cytostatic agents, either alone or in 
combination, include alkylating agents (cyclophosphamide/ifosfamide), platinum-based agents (cisplatin/carboplatin), etoposide, adriamycin and vinca-alkaloids.

Clinical data on the survival outcomes of adult patients (defined as 20 years of age and older) with NB are scarce due to the rarity of the disease. Small, single institution reports have described worse outcomes for adults than pediatric patients.

Currently, there are no standard treatment guidelines for patients with adult NB. In patients with metastatic disease and at late stages of disease, multimodal therapy should include surgical resection, radiotherapy, and outpatient chemotherapy. Follow-up of patients with NB should include metabolic imaging studies and tumor marker determination. The collection and evaluation of data on adult patients with this rare tumor are warranted in order to optimize treatment strategies. Whether more intensive treatment or myeloablative therapy with stem cell support will improve the outcome of NB in adults is unclear.

\section{Radiotherapy}

Radiotherapy has no role in low-risk NB. Stage IV NB is not a localized disease and systemic treatment is required. The efficacy of radiotherapy in the high-dose chemotherapeutical protocols has not been established yet. The efficacy of RT should be weighted against the potential late effects (growth retardation, scoliosis, secondary malignancy) and the benefit-hazard ratio should be considered. The role of radiotherapy in high risk is more marked compared to the moderate risk group.

\section{Surgery}

Surgical intervention is significantly involved in the treatment of $\mathrm{NB}$, and complete excision of the tumor or minimization of the tumor to the highest extent possible has a favorable effect on the prognosis [13].

\section{Immunology and Neuroblastoma}

Based on the fact that the human immune system may be deficient in recognizing and destroying the NB cells, active and passive immunotherapies are being developed. For passive immunotherapy, anti-GD2 monoclonal antibodies and genetically modified T cells are used. For active immunotherapy, IL-2, modified tumor cell vaccines, dendritic cell vaccines, DNA vaccines and monoclonal antibodies with non-specific signaling (antiCTLA-4, anti-CD40) are used. Treatment protocols based on immunotherapy may also be used $[14,15]$.

The other novel therapeutical approaches in the treatment of NB include the use of retinoic acid, anti-N-myc therapies and angiogenesis-regulating therapies and the use of histone deacetylase inhibitors. Retinoic acid derivatives stimulate the differentiation of neuroblasts, thereby contributing to the treatment. N-myc is an intracellular component. When silencing RNA (siRNA) is placed in the structure of the N-myc, the cell differentiation and programmed cell death increase and the tumor growth stops. The 
presence of the $\mathrm{N}$-myc recipients on the cell surface has been demonstrated and an increase in their number achieved by stimulation has been observed to lead to an increased programmed cell death and an increased responsiveness in these cells against hydroxyurea. Anti-VEGF antibodies may provide a narrowing in the tumor by decreasing vascularization. In malignancies, DNA methylation and histone acetylation is increased. Histone deacetylase inhibitors (HDAC1) interfere with the development of the tumor by preventing the occurrence of this effect.

Patient-specific therapeutical strategies are being developed to increase the survival rates, reduce the late effects and increase the quality of life in NB $[16,17]$.

\section{Disclosure Statement}

There is no potential conflict of interest.

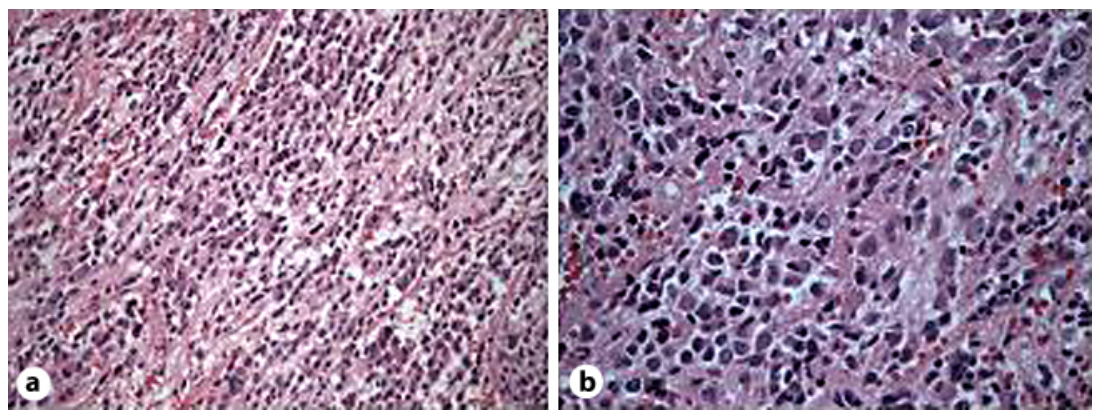

Fig. 1. a, b Nuclear vesiculation, nucleolar prominence, extension and eosinophilic amphophilic features observed in the cytoplasm. 


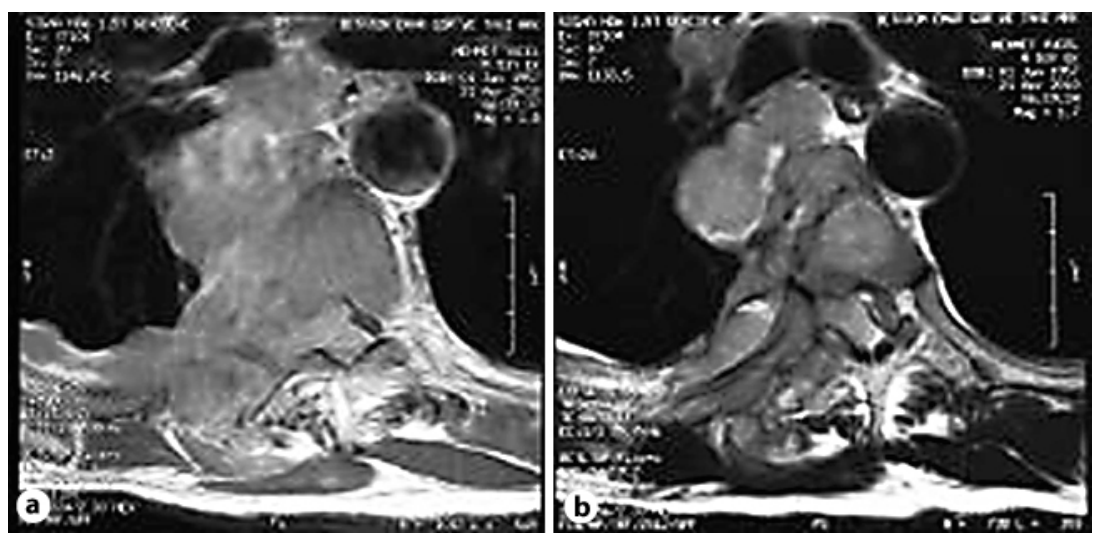

Fig. 2. a, b On the T1- and T2-weighted axial cross-sections, at the T4, T5 and T6 levels, the tumor extends from the right neural foramina to the spinal duct and creates a marked compression on the spinal cord from the right posterolateral section with a $5.5-\mathrm{cm}$ long component. In addition, there are diffuse lymphadenomegalies in the paravertebral region.

\section{References}

${ }_{1}$ McLean TW, Iskandar SS, Shimada H, Hall MC: Neuroblastoma in an adult. Urology 2004;64:1232.

$\checkmark 2$ Armstrong EA, Harwood-Nash DC, Ritz CR, et al: CT of neuroblastomas and ganglioneuromas in children. AJR 1982;139:571-576.

3 Ribet ME, Cardot GR: Neurogenic tumors of the thorax. Ann Thorac Surg 1994;58:1091-1095.

4 Reed JC, Hallet KK, Feigin DS: Neural tumors of the thorax: subject review from the AFIP. Radiology 1978;126:9-17.

$\checkmark 5$ Conte M, Parodi S, De Bernardi B, Milanaccio C, Mazzocco K, Angelini P, et al: Neuroblastoma in adolescents: the Italian experience. Cancer 2006;106:1409-1417.

6 Coldman AJ, et al: Neuroblastoma: influence of age at diagnosis, stage, tumor site, and sex on prognosis. Cancer 1980;46:1896-1901.

7 Gray JC, Kohler JA: Immunotherapy for neuroblastoma: turning promise into reality. Pediatr Blood Cancer 2009;53:931-940.

-8 Franke A, Jentsch-Ullrich K, Schalk E, Mohren M, Koenigsmann M, Buhtz P: Metastatic adrenal neuroblastoma in an adult. Onkologie 2005;28:353-355.

-9 Conte M, De Bernardi B, Milanaccio C, Michelazzi A, Rizzo A, Montobbio G, et al: Malignant neuroblastic tumors in adolescents. Cancer Lett 2005;228:271-274.

${ }_{10}$ Kushner BH, Kramer K, LaQuaglia MP, et al: Neuroblastoma in adolescents and adults: the Memorial SloanKettering experience. Med Pediatr Oncol 2003;41:508-515.

11 Tang CK, Hajdu SI: Neuroblastoma in adolescence and adulthood. NY State J Med 1975;75:1434-1438.

-12 Kushner BH, Kramer K, Modak S, Qin LX, Cheung NK: Differential impact of high-dose cyclophosphamide, topotecan, and vincristine in clinical subsets of patients with chemoresistant neuroblastoma. Cancer 2010;116:3054-3060.

13 Ishola TA, Chung DH: Neuroblastoma. Surg Oncol 2007;16:149-156.

14 Navid F, Armstrong M, Barfield RC: Immune therapies for neuroblastoma. Cancer Biol Ther 2009;8:874-882.

15 Schulte JH, Horn S, Schlierf S, et al: MicroRNAs in the pathogenesis of neuroblastoma. Cancer Lett 2009;274:10-15.

16 Stallings RL: MicroRNA involvement in the pathogenesis of neuroblastoma: potential for microRNA mediated therapeutics. Curr Pharm Des 2009;15:456-462.

17 Karnak I: The role of pediatric surgeon in the modern pediatric oncological approach: update to 2010. Turk Arch Ped 2010;45(suppl):110-114. 